\title{
Aerobic Bacteriological Profile and its Antimicrobial Sensitivity Pattern From Blood Culture Specimens in A Tertiary Care Hospital.
}

\author{
Santosh A. Kotgire*, Sunil Hatkar S \\ Department of Microbiology, Indian Institute of Medical Sciences, Jalna, Maharashtra, India
}

\begin{abstract}
Background :Blood stream infections (BSIs) are important cause of mortality and morbidity and are among most common health-associated infections.Blood stream infection constitutes one the most serious situations with increased cost of care, morbidity, mortality and thus, timely detection and identification of blood stream pathogen is important.Sothe present study was undertaken to describe aerobic bacteriological profile and its antibiotic sensitivity pattern from blood culture specimen in a tertiary care setting.

Method: The study was carried out in the Department of Microbiology, Indian Institute of Medical Sciences, Jalna, Maharashtra for a period of 8 months (July 2015 to February 2016). A total 1920 samples were evaluated from clinically suspected cases of bacteremia. Blood was collected depending upon age group with aseptic precaution and inoculated on brain heart infusion broth (BHIB). Subculture were made on blood agar and Mac-conkey agar plates. Organisms were identified and antibiotic sensitivity test of isolates were performed.

Result : During 8 month study period out of 1920 blood culture, 369(19.21\%) yielded growth of different organisms. Out of this 369 organisms gram positive bacteria 199 (53.9\%) were isolated more often than gram negative bacteria 170 (46.1\%). Staphylococcus aureus $49.05 \%$ was leading pathogens isolated followed by enterobactericae group $21.00 \%$ (Escherichia coli and Klebsiella spp) and Salmonella typhi \& S. Paratyphi A $13.82 \%$.
\end{abstract}

Conclusion: This study provides information on antibiotic sensitivity pattern of blood isolates which may be useful to guide clinicians to initiate empiric therapy and will help in formulation of antibiotic therapy strategy in this part of country.

\section{Keywords: Blood Culture, Antimicrobial Sensitivity Pattern, Bacteremia.}

\section{Introduction}

Blood stream infections (BSIs) are important cause of mortality and morbidity and are among most common health-associated infections ${ }^{[1]}$.Bacteremia signifies the presence of bacteria in the blood stream ${ }^{[1]}$. Bacteremia may be transient, continuous or intermittent. Micro-organisms present in the circulating blood are a threat to every organ in the body ${ }^{[1]}$.

It can have serious consequences like shock, multiple organ failure, disseminated intravascular coagulation, etc. thus, the blood stream infection constitutes one the most serious situations with increased cost of care, morbidity, mortality and, as a result,timely detection and identification of blood stream pathogen is important. Blood culture plays an integral role in the evaluation of sepsis ${ }^{[1,2]}$.

Increasing antimicrobial resistance is worldwide concern. The prevalence of resistance in both out-patients and hospitalised patients with septicaemia is increasing, and its varies in accordance with geographical and regional location. In almost all cases, antimicrobial therapy is initiated empirically before the results of blood culture are available ${ }^{[3]}$.
Selecting appropriate antimicrobial for treating BSI is multifaceted, including possible cause and source of infection, in vitro activity of drug according to microbiological susceptibility testing results, pharmacokinetics and adverse effects of the drug ${ }^{[2]}$.

However, before considering these aspects, the choice of antibiotic mainly relies on knowledge of the pathogen likely involved.Monitoring and analysing the antimicrobial suscepitibility pattern of most frequently isolated microorganisms according to local epidemiology which helps clinicians to choose empirical therapies and develop rational prescription policy for antibiotics ${ }^{[4,5]}$.

Therefore, the present study was undertaken to describe aerobic bacteriological profile and its antibiotic sensitivity pattern from blood culture specimen in a tertiary care setting to guide clinicians to initiate empiric antibiotic therapy and to formulate antibiotic policy.

\section{Materials and Methods}

Study Design: The present study is prospective type of study and was carried out at Department of Microbiology, 
Indian Institute of Medical Sciences Badnapur, Jalna, Maharshtra after approval from institutional ethics committee.

Sample Size: The study was carried out during the period of July 2015-February 2016; a total 1920 samples were evaluated from clinically suspected cases of bacteremia.

Inclusion Criteria: All patients with unexplained fever/ undiagnosed fever, whose blood culture specimens were sent to department of microbiology were included in the study.

Exclusion Criteria: Contaminated, duplicate and repeat specimens from patients were excluded from study.

Collection of Sample and Processing : In adults $5 \mathrm{ml}$ of blood for culture was drawn in sterile syringe after skin preparation by a two step process with $70 \%$ alcohol andpovidone iodine application and then dried for $1 \mathrm{~min}$. Blood collected was aseptically incubated into blood culture bottle containing $50 \mathrm{ml}$ of Brain Heart Infusion Broth (BHIB). In paediatric cases $1-2 \mathrm{ml}$ of blood was inoculated in $5-10 \mathrm{ml}$ of BHIB.These bottles will be incubated at $37^{\circ} \mathrm{C}$ temperature under aerobic conditions in the incubator maximum for 7 days. Subculture will be made on Blood agar and MacConkey's agar daily from 1st to 7 th day. However if the growth was observed further sub cultures were not done. Growth was processed according to standard microbiological techniques which includes Gram staining, colony characteristics and biochemical properties described in WC. Koneman's Colour Atlas and text book of Diagnostic Microbiologyand Bailey and Scott's Diagnostic Microbiology ${ }^{[6,7]}$. Blood culture broth which showed no microbial growth after 7 days were reported as culture negative.

Antimicrobial Sensitivity Testing Criteria for antimicrobial sensitivity testing used was carried out as per Clinical Laboratory standard institute (CLSI) ${ }^{[8]}$.Antimicrobial sensitivity testing was done on Muller Hinton Agar (MHA) by Kirby Bauer disc diffusion method. Commercially available discs (Hi-media) were be used. Concentration of discs used were Erythromycin $(15 \mathrm{mcg})$, Vancomycin (30mcg), Co-trimoxazole (25mcg), Ciprofloxacin (5mcg), Linezolid (30mcg), Ampicillin (30mcg), Piperacillin+Tazobactum (100/10mcg), Ceftazidime (30 $\mathrm{mcg}$ ), Amikacin (30 mcg), Ofloxacin (5mcg), Gentamicin (10mcg) \& high level (30mcg), Furazolidone(300mcg), Azetronam(30mcg), Chloramphenicol (30mcg), Ceftriaxone (30mcg), Amoxicillin (20mcg) and Imipenem (10mcg).

Methicillin resistance in Staphylococcus aureus (MRSA) was tested using Muller Hinton Agar with Cefoxitin disc (30mcg) by Kirby-bauerdisc diffusion methods as per
CLSI guidelines ${ }^{[8]}$. Suspected extended- spectrum beta lactamases (ESBLs) producing Enterobactericae were confirmed by double disk synergy test as as per CLSI guidelines $^{[8]}$.

Staphylococcus aureus (ATCC 25923), E. coli (ATCC 25922) and $P$. aeruoginosa (ATCC 27853) were used as quality control throughout the study for culture and antimicrobial susceptibility testing.

Statistical analysis The results were expressed as percentages for analysis of various epidemiological details and for analysing the distribution of different bacterial isolates and their sensitivity pattern. Microsoft excel was used for the interpretation of these results.

\section{Results}

During 8 month study period 1920 blood culture were analysed. 369(19.21\%) yielded growth of different organisms. Of all isolates 291(76.8\%) were isolated from hospitalised patients while $78(23.2 \%)$ were from those who attended out-patients department. Majority of the patients were males $285(77.23 \%)$; male to female ratio was $3.4: 1$.

Out of this 369 organisms, gram positive bacteria 199 $(53.9 \%)$ were isolated more often than gram negative bacteria 170 (46.1\%). In our study Staphylococcus aureus $49.05 \%$ was leading pathogens isolated followed by Enterobactericae group 21.00\% (Escherichia coli and Klebsiellaspp) and Salmonella typhi \& S. paratyphi A $13.82 \%$.

The detailed microbiological data of pathogens and their antimicrobial susceptibility causing blood stream infection is shown in table $1,2,3 \& 4$

\section{Discussion}

The varying microbiological pattern of bacteremia/ septicaemia warrants the need for an ongoing review of causative organisms and their antimicrobial susceptibility pattern.

Out of 1920 suspected cases of bacteremia, in our study, 369 were culture positive with blood culture positivity rate of $19.21 \%$. Similar positivity rates were reported by other studies $^{[9,10]}$.

Our study highlights that gram positive septicaemia was encountered in $53.9 \%$ culture positive cases which was in concordance with studies done by China et al; and Gupta et.al; which shows increase incidence of gram positive bacteria especially Staphylococcus aureus in producing BSIs $^{[11,12]}$. 
Table 1 Distribution of isolates

\begin{tabular}{|l|c|c|c|c|c|c|c|}
\hline Organisms & OPD & Med & Paeds & ICU & Surg & Others & Total $(\mathbf{n}=369)$ \\
\hline Staphylococcus aureus & 25 & 36 & 29 & 15 & 20 & 56 & 181 \\
\hline Salmonella typhi and S. paratyphi A & 16 & 12 & 09 & 03 & 01 & 10 & 51 \\
\hline E.coli & 08 & 18 & 10 & 08 & 0 & 02 & 46 \\
\hline Klebsiellaspp & 05 & 10 & 05 & 07 & 02 & 02 & 31 \\
\hline Pseudomonas aeruginosa & 04 & 03 & 05 & 10 & 04 & 03 & 25 \\
\hline Acinetobacterspp & 02 & 02 & 02 & 07 & 03 & 01 & 17 \\
\hline Enterococci spp & 06 & 05 & 05 & 01 & 0 & 01 & 18 \\
\hline
\end{tabular}

OPD-out patient department, Med-medicine ward, Paeds-paediatric ward, ICU-intensive care unit, Surg-surgery ward, Othersobstetrics \&gynaecology ward, orthopaedic ward, etc

Table 2:Distribution of organisms isolated from blood culture.

\begin{tabular}{|l|c|c|}
\hline Name of organism & Numbers (n=369) & Prevalence\% \\
\hline Staphylococcus aureus & 181 & 49.05 \\
\hline E.coli & 46 & 12.46 \\
\hline Klebsiellaspp & 31 & 8.54 \\
\hline Salmonella typhi/Para typhi A & 51 & 13.82 \\
\hline Pseudomonas aeruginosa & 25 & 6.77 \\
\hline Enterococci spp & 18 & 4.87 \\
\hline Acinetobacterspp & 17 & 4.60 \\
\hline Total & 369 & 100.00 \\
\hline
\end{tabular}

Table 3: Antimicrobial sensitivity pattern of Gram positive organisms.

\begin{tabular}{|l|c|c|}
\hline Antibiotics & Staphylococcus aureus(n=181) & Enterococci spp (n=18) \\
\hline Penicillin & $41.4 \%(75)$ & $44.44 \%(08)$ \\
\hline Cotrimaxozole & $49.72 \%(90)$ & Not tested \\
\hline Ciprofloxacin & $49.72 \%(90)$ & Not tested \\
\hline Erythromycin & $55.24 \%(100)$ & Not tested \\
\hline Gentamicin & $70.16 \%(127)$ & Not tested \\
\hline Cefoxitin & $77.34 \%(140)$ & $100 \%(18)$ \\
\hline Vancomycin & $100 \%(181)$ & $100 \%(18)$ \\
\hline Linezolid & $100 \%(181)$ & $83.33 \%(15)$ \\
\hline Gentamicin (high level) & Not tested & $88.88 \%(16)$ \\
\hline Ampicillin & Not tested & \\
\hline
\end{tabular}

Table 4: Antimicrobial sensitivity pattern of Gram negative organisms

\begin{tabular}{|l|c|c|c|c|c|}
\hline Antibiotics & E.coli(n=46) & Klebsiellaspp(n=31) & $\begin{array}{c}\text { Salmonella typhi/ } \\
\text { paratyphiA(n=51) }\end{array}$ & $\begin{array}{c}\text { Pseudomonas } \\
\text { aeruginosa(n=25) }\end{array}$ & $\begin{array}{c}\text { Acinetobacterspp } \\
(\mathrm{n}=17)\end{array}$ \\
\hline Cotrimaxozole & $43.47 \%(20)$ & $48.38 \%(15)$ & $80.39 \%(41)$ & $48.00 \%(12)$ & $52.94 \%(09)$ \\
\hline Ciprofloxacin & $50.00 \%(23)$ & $54.83 \%(17)$ & Not tested & $76.00 \%(19)$ & $70.58 \%(12)$ \\
\hline Ceftazidime & $54.34 \%(25)$ & $58.06 \%(18)$ & Not tested & $80.00 \%(20)$ & $76.47 \%(13)$ \\
\hline Amikacin & $65.21 \%(30)$ & $64.51 \%(20)$ & Not tested & $88.00 \%(22)$ & $82.35 \%(14)$ \\
\hline Gentamicin & $82.60 \%(38)$ & $80.64 \%(25)$ & Not tested & $92 \%(23)$ & $58.82 \%(10)$ \\
\hline Ampicillin-sulbactam & $89.13 \%(41)$ & $93.54 \%(29)$ & $88.23 \%(45)$ & Not tested & Not tested \\
\hline
\end{tabular}




\begin{tabular}{|l|c|c|c|c|c|}
\hline Antibiotics & E.coli(n=46) & Klebsiellaspp(n=31) & $\begin{array}{c}\text { Salmonella typhi/ } \\
\text { paratyphiA(n=51) }\end{array}$ & $\begin{array}{c}\text { Pseudomonas } \\
\text { aeruginosa(n=25) }\end{array}$ & $\begin{array}{c}\text { Acinetobacterspp } \\
(\mathrm{n}=17)\end{array}$ \\
\hline Azetronam & $73.91 \%(34)$ & $83.87 \%(26)$ & Not tested & $56.00 \%(14)$ & $47.05 \%(08)$ \\
\hline Pipercillin-tazobactam & Not tested & Not tested & Not tested & $100 \%(25)$ & $100 \%(17)$ \\
\hline Chloramphenicol & Not tested & Not tested & $76.47 \%(39)$ & Not tested & Not tested \\
\hline Furazolidone & Not tested & Not tested & $62.74 \%(32)$ & Not tested & Not tested \\
\hline Imipenem & $100 \%(46)$ & $100 \%(31)$ & Not tested & $100 \%(25)$ & $100 \%(17)$ \\
\hline Ceftriaxone & Not tested & Not tested & $100 \%(51)$ & Not tested & Not tested \\
\hline Amoxicillin & Not tested & Not tested & $82.35 \%(42)$ & Not tested & Not tested \\
\hline
\end{tabular}

Gram negative septicaemia was encountered in $46.1 \%$ of culture positive cases which is lower as compared to other studies which had reported increased incidence of gram negative bacteria ranging from $50 \%$ to $78 \% \%^{[12,13]}$. Commonest gram negative bacteria isolated in our study was E.coli , Klebsiella spp and followed by Salmonella spp and non-fermenters (Pseudomonas and Acinetobacter spp) which was in concordance with other studies carried out in different parts of India ${ }^{[14,15]}$.

The gram positive organism especially Staphylococcus aureus showed $41.4 \%$ and $55.24 \%$ sensitivity to penicillin and erythromycin respectively but were 100 sensitive to Vancomycin and Linezolid similar sensitivity pattern were seen in other studies ${ }^{[11,14,15]}$. 41(22.65\%) were detected as methicillin resistant Staphylococcus aureus (MRSA). Studies by Indian researchers ${ }^{[16]}$, reported a similar prevalence of $41 \%$ MRSA. Our study also point out increase isolation of Enterococci spp as many studies had not isolated any single case of Enterococci spp except for few ${ }^{[17,18]}$. All Enterococci Spp were $100 \%$ sensitive to Vancomycin and showed $88.88 \%$ and $83.33 \%$ sensitivity to Ampicillin and Gentamicin (high level) respectively.

Most of gram negative bacteria especially Enterobactericae (except Salmonella spp )showed 100 sensitivity to imipenem and high resistance to ciprofloxacin and ceftazidime, similar trends has been reported by an Indian study ${ }^{[19]}$ and many other researchers ${ }^{[11,12]}$ highlighting higher resistance to third generation cephalosporins. Our study found out around 30\% Enterobactericae isolates were ESBL producers which is alarmingly on higher side as compared with other studies ${ }^{[13]}$.

While member of nonfermenter (Pseudomonas and Acinetobacter spp) shown $100 \%$ sensitivity to Imipenem and Pipercillin-tazobactam this results are comparable to work done at other centers ${ }^{[19,20]}$ and also revealed better sensitivity to third generation Cephalosporin and Gentamicin as compared with other studies ${ }^{[18,20]}$.

\section{Conclusion}

The detection, identification and susceptibility testing of causative species of bacteria are essential for proper treatment and better prognosis of patient in case of BSIs. Blood culture still remains as one of the most important microbiological investigation available to clinicians for diagnsosis of BSIs. Gram positive organisms were predominant in our setup for producing BSIs with higher incidence of MRSA. Our study also showed alarmingly higher incidence of ESBL producing Enterobacterciae group which points towards judicious uses of third generation Cephalosporins.

\section{References}

1. Campos JM, McNamara AM, Howard BJ. Specimen collection and Processing. Clinical and Pathogenic Microbiology.Mosby; 1994:195-213.

2. Shanson DC. Blood culture technique: current controversies. J Antimicrob Chemother. 1990; 25:17-29.

3. Meremkwer MM, Nwachukwu CE, et al. Bacterial isolates from blood cultures of children with suspected septicaemia in Calabar, Nigeria. BMC Infect Dis. 2005; 5:110.

4. Nwadioha SI, Nwokedi EOP, Kashibu E, et al. A review of bacterial isolates in blood cultures of children with septicaemia in a Nigerian tertiary Hospital. Afr J Microbiol Res. 2010; 4:222.

5. Baltimore RS, Bogue CW. Infections Disease - Foci of Infection. In: Burg FD, Polin RA, Ingelfinger JR, Gershon AA, Editors. Gellis\& Karan's current pediatric therapy. 17th Edition. USA. Elsevier Science;2002.39-256.

6. Koneman EW, Allen SD, Janda WM, Schreckember PC, Winn WC. Koneman's Colour Atlas and text book of Diagnostic Microbiology. 6th edition. Newyork: Lippincott: 2006.97-99.

7. Forbes BA, Sahm DF, Weissfeld AS. In: Bailey and Scott's Diagnostic Microbiology. Mosby Elsevier;2007: 779.

8. CLSI - Clinical and Laboratory Standards Institute 2016. Performance standards for antimicrobial susceptibility testing. Twenty-second informational supplement. Wayne, PA, USA. CLSI;2016. 
9. Nimri L.F, Ravashdeh M, Meqdam M.M. Bacteremia in Children: Etiologic Agents, Focal Sites, And Risk Factors. Jr of tropical pediatrics. 2001;356:60.

10. Prabhu K, Bhat S, Rao S. Bacteriologic profile and antibiogram of blood culture isolates in a pediatric care unit. J Lab Physicians. 2010;2:85-8.

11. Chinaand D, Gupta V. Bacteriological profile and antimicrobial susceptibility pattern of blood isolates from a tertiary care hospital in North India. International Journal of Pharmaceutical Research and Bioscience.2013;2:24-35.

12. Gupta, A., Sharma, S., Arora, A. et al. Changing Trends of in Vitro Antimicrobial Resistance Patterns in Blood Isolates in a Tertiary Care Hospital over a Period of 4 Years. Indian Journal of Medical Sciences 2010. 64 (11); 485-92.

13. Chaudhary U, Aggarwal R. Extended spectrum-lactamases (ESBL) - An emerging threat to clinical therapeutics. Indian J Med Microbiol. 2004;22:75-80

14. . Noyal MJC, Menezes GA, Harish BN, et al. Simple screening test for detection of carbapenamases in clinical isolates of nonfermentative gram negative bacilli. Indian $\mathrm{J}$ Med Res. 2009; 29: 707-12.

15. Alam, MS. Pillai, P. K. Kapur, et al Resistant Patterns of Bacteria Isolated from Bloodstream Infections at a
University Hospital in Delhi. J Pharm Bioallied Sci 2011; 3 (4) $525-30$.

16. Datta, S., Wattal, C., Goel, N., et al A Ten Year Analysis of Multi-Drug Resistant Blood Stream Infections Caused by Escherichia Coli \&Klebsiella Pneumoniae in A Tertiary Care Hospital. Indian Journal of Medical Research. 2011;135 (6) 907-12.

17. Kamga HLF, Njunda AL, Nde PF. Prevalence of septicaemia and antibiotic sensitivity pattern of bacterial isolates at the University Teaching Hospital, Yaoundae, Cameroon. African Journal of Clinical and Experimental Microbiology 2010:12(1):198-201

18. Barati, M. Taher, MT. Abasi, et al Bacteriological Profile and Antimicrobial Resistance of Blood Culture Isolates.Iranian Journal of Clinical Infectious Diseases.2009:2 (4) 87-95.

19. Garg A, Anupurba S, Garg J. Bacteriological profile and antimicrobial resistance of blood culture isolates from a university hospital .Journal of Indian Academy of Clinical Medicine.2007;8(2):139-143

20. Anbumani N, Kalyani J, Mallika M. Distribution and antimicrobial susceptibility of bacteria isolated from blood cultures of hospitalized patients in a tertiary care hospital,' Indian Journal for the Practicing Doctor.2005:5(2): 1-7.

*Corresponding author:

Santosh A. Kotgire, Department of Microbiology, Indian Institute of Medical Sciences, Jalna, Maharashtra, India

Phone: +91 9922867558

Email: santosh_kots2001@yahoo.com

Date of Submission : 25.07.2016

Date of Acceptance : 21.11.2016

Financial or other Competing Interests: None.

Date of Publication : 04.02.2017 